\title{
Crescimento de plantas de Salvia officinalis sob ação de reguladores de crescimento vegetal
}

\author{
Growth of Salvia officinalis plants under action of plant growth regulators
}

\author{
Juliana Aparecida Povh' ${ }^{\text {Elizabeth Orika Ono }}{ }^{\text {II }}$
}

\section{RESUMO}

O objetivo deste trabalho foi estimar os parâmetros da análise de crescimento em função de diferentes reguladores vegetais aplicados na parte aérea de plantas de Salvia officinalis L. Para tanto,o experimento foi instalado em casa de vegetação do Departamento de Botânica, Instituto de Biociências, da Universidade Estadual Paulista, Botucatu, SP. Os tratamentos consistiram na pulverização da solução de $100 \mathrm{mg} \mathrm{L}^{-1}$ de ácido giberélico $\left(G A_{3}\right) ; 100 \mathrm{mg} \mathrm{L}^{-1}$ de benzilaminopurina (BAP); $100 \mathrm{mg} \mathrm{L}^{-1}$ de ácido 2-cloroetil-fosfônico (ethephon);

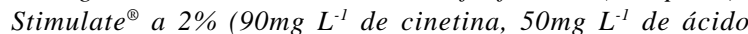
giberélico e $50 \mathrm{mg}^{-1}$ de ácido indolilbutírico) e água (testemunha). As aplicações foram realizadas em três épocas, aos 15, 25 e 35 dias após o transplante (d.a.t.) e o crescimento foi avaliado em cinco épocas de coletas a intervalos de 21 dias, sendo a primeira realizada aos 47 (d.a.t.). Foram determinados os parâmetros fisiológicos da análise de crescimento: razão de área foliar (RAF), área foliar específica (AFE), taxa assimilatória líquida (TAL) e taxa de crescimento relativo (TCR). Os resultados mostram que os reguladores de crescimento vegetal influenciaram os parâmetros fisiológicos da análise de crescimento. As plantas tratadas com BAP apresentaram maiores valores de RAF aos 47 d.a.t., já as plantas tratadas com GA, a TAL apresentou aumento até o 131 d.a.t. A TCR é decrescente para todos os tratamentos com reguladores de crescimento vegetal testados e a testemunha.

Palavras-chave: Lamiaceae, ácido giberélico, benzilaminopurina, área foliar específica.

\section{ABSTRACT}

The objective of this study was to estimate the effects of different plant regulators on the index growth analysis application of sage plants. An experiment was conducted in a greenhouse with controlled temperature and relative humidity, at the Departmento de Botânica, Instituto de Biociências, Universidade Estadual Paulista, UNESP, Botucatu, SP, Brazil. The experimental design was completely randomized, with five treatments containing three replicates. Treatments consisted of the spraying of the solution of $100 \mathrm{mg} \mathrm{L}^{-1}$ of gibberellic acid $\left(G A_{3}\right) ; 100 \mathrm{mg} \mathrm{L}^{-1}$ of benzylaminopurine (BAP); $100 \mathrm{mg} \mathrm{L}^{-1}$ of 2-chloroethyl phosphonic acid (ethephon); Stimulate ${ }^{\circledR}$ at $2 \%$ (90mg $\mathrm{L}^{-1}$ of kinetin, $50 \mathrm{mg} \mathrm{L}^{-1}$ of gibberellic acid and $50 \mathrm{mg} \mathrm{L}^{-1}$ of indolylbutyric acid) and water (control). Applications of plant growth regulators were performed in 3 times, at 15, 25, and 35 days after transplanting, and growth was evaluated in five successive harvests at 21-day intervals, performed at 47, 68, 89, 110, and 131 days after transplanting. The following growth index were determined: leaf area ratio (LAR), specific leaf area (SLA), net assimilation rate (NAR), and relative growth rate (RGR). These results suggest that the plant growth regulators influence the index growth analysis. The plants treated with $B A P$ presented increase values of LAR to the 47 days after transplanting. The plants treated with GA to NAR presented increase until the 131 days after transplanting. The RGR is decreasing for all the treatments with vegetal regulators tested and the control.

Key words: Lamiaceae, gibberellic acid, benzylaminopurine, specific leaf area.

\section{INTRODUÇÃO}

Salvia officinalis L., pertencente à família Lamiaceae, é originária do mediterrâneo e aclimatada, principalmente, na região Sul do Brasil. É considerada uma planta aromática e com propriedades medicinais (BARICEVIC \& BARTOL, 2000). Entre suas propriedades terapêuticas, destacam-se as emenagoga,

IPrograma de Pós-graduaçao em Ciências Biológicas (Botânica), Universidade Estadual Paulista (UNESP), CP 510, 18618-000,

Botucatu, SP, Brasil. E-mail: japovh@hotmail.com. Autor para correspondência.

IIDepartamento de Botânica, Instituto de Biociências, UNESP, Botucatu, SP, Brasil. 
diaforética, germicida, antiinflamatória, antioxidante e adstringente (EVANS 1991; HERTWIG 1991; COSTA 1994). Além da sua utilização na medicina tradicional, possui grande importância econômica para a indústria farmacêutica, cosmética e alimentícia, sendo o cultivo dessa espécie de grande importância econômica (CUVELIER et al., 1996; MARTINS et al., 1998).

$\mathrm{O}$ valor comercial de plantas medicinais e aromáticas tem refletido qualitativa e quantitativamente na produção destas espécies. O uso de reguladores de crescimento vegetal está sendo muito usado na agricultura devido a suas influências positivas na quantidade e na qualidade de produção. Porém, pouco se conhece sobre os efeitos destas substâncias em plantas medicinais e aromáticas.

Alguns estudos têm mostrado que essas substâncias têm sido muito utilizadas em plantas medicinais visando à promoção do crescimento e ao aumento na produtividade (SHUKLA \& FAROOQI, 1990). BARREIRO et al. (2006) relatam para plantas de Ocimum basilicum $\mathbf{L}$, pertencente à família Lamiaceae, o efeito de alguns reguladores vegetais no crescimento vegetal, destacando que os índices fisiológicos da análise de crescimento foram influenciados positivamente por estas substâncias, principalmente pela cinetina. Contudo, mais estudos são necessários para melhorar a produção comercial de plantas medicinais. A análise de crescimento neste trabalho será usada como uma ferramenta importante para avaliar a eficiência dessas substâncias no crescimento dessa espécie.

A análise de crescimento é um método que descreve as condições morfofisiológicas da planta em diferentes intervalos de tempo entre duas amostragens sucessivas (MAGALHÃES, 1986). Segundo BENINCASA (2003), esse tipo de análise baseia-se fundamentalmente no fato de que cerca de $90 \%$, em média, da matéria seca acumulada pelas plantas, ao longo do seu crescimento, resultam da atividade fotossintética e o restante resulta da absorção de nutrientes minerais.

Apesar de alguns estudos demonstrarem que a aplicação de reguladores vegetais pode aumentar a produtividade vegetal, a fisiologia de plantas medicinais é carente de informações sobre o crescimento e o desenvolvimento de várias espécies nativas ou cultivadas no Brasil. Desse modo, o presente estudo tem como objetivo avaliar os parâmetros fisiológicos de plantas de Salvia officinalis L. tratadas com diferentes reguladores de crescimento vegetal.

\section{MATERIAL E MÉTODOS}

O experimento foi conduzido em casa de vegetação no Departamento de Botânica, Instituto de Biociências, da Universidade Estadual Paulista, UNESP, Botucatu, SP. As plantas foram obtidas a partir de sementes já tratadas com thiram (dissulfeto tetrametil - tiuram) previamente germinadas em câmara de germinação sob luz branca constante à temperatura de $25^{\circ} \mathrm{C}$. Plântulas com 21 dias foram transferidas para vasos, duas por vaso, com capacidade de quatro litros, com terra corrigida (Calagem - 4,0g vaso ${ }^{-1}$ de calcáreo dolomítico) e adubada (400 $\mathrm{mg}^{\text {vaso }}{ }^{-1}$ de superfosfato simples, 6,8g vaso ${ }^{-1}$ da fórmula 4-14-8 e $10 \%$ em volume do vaso de matéria orgânica, esterco de vaca). As plantas foram mantidas em casa de vegetação e irrigadas sempre que necessário. A temperatura máxima dentro da casa de vegetação, durante a condução do experimento, foi $26^{\circ} \mathrm{C}$ e a mínima foi $12^{\circ} \mathrm{C}$.

Foram estabelecidos cinco tratamentos com quatro reguladores vegetais diferentes, conforme descrito: 1 - testemunha (água); 2 - ácido giberélico $\left(\mathrm{GA}_{3}\right)$ a $100 \mathrm{mg} \mathrm{L}^{-1} ; 3$ - benzilaminopurina (BAP) a 100mg L $\mathrm{m}^{-1} ; 4$ - ácido 2 cloroetil - fosfônico (ethephon) a 100mg L-1; 5 - ácido giberélico $\left(\mathrm{GA}_{3}\right)$ + cinetina + ácido ildolilbutírico (IBA) a 2\%.

$\mathrm{O}$ ácido giberélico $\left(\mathrm{GA}_{3}\right)$ foi utilizado na forma do produto comercial Pro-Gibb, contendo $10 \%$ de $\mathrm{GA}_{3}$, da Abbott Laboratórios do Brasil Ltda.; a benzilaminopurina (BAP) foi utilizada na forma do produto p.a. fabricado pela Sigma; o ácido 2 cloroetilfosfônico (ethephon) foi utilizado na forma do produto comercial ETHREL, contendo $240 \mathrm{~g}$ de ácido 2 cloroetilfosfônico, fabricado pela Rhodia Agro S.A., a mistura de ácido giberélico $\left(\mathrm{GA}_{3}\right)+$ cinetina + ácido ildolilbutírico (IBA) foi utilizada na fórmula comercial Stimulate ${ }^{\circledR}$ contendo $90 \mathrm{mg} \mathrm{L}^{-1}$ de cinetina, 50 $\mathrm{mg} \mathrm{L}^{-1}$ de IBA e 50 $\mathrm{mg} \mathrm{L}^{-1}$ de $\mathrm{GA}_{3}$, fabricado pela Stoller S.A.

As pulverizações foram realizadas com pulverizador manual com ponta de $110^{\circ} 02$ sob pressão constante de 40 libras; na solução contendo regulador do crescimento vegetal, foi adicionado espalhante adesivo não-iônico alquil-fenol-poliglicoléter, ou seja, o produto comercial Extravon na dose de $0,5 \mathrm{~mL} \mathrm{~L}^{-1}$ de solução, fabricado pela Ciba - Geigy Química S/A. Foram realizadas três aplicações, a primeira 15 dias após o replantio das plantas nos vasos, onde as plantas já estavam estabilizadas. A segunda e a terceira aplicação foram realizadas com 10 e 20 dias após a primeira, respectivamente; em cada época de aplicação, foram utilizados 310mL de solução de cada tratamento.

O delineamento experimental utilizado foi o inteiramente casualizado em esquema fatorial $5 \times 5$, cinco 
tratamentos e cinco épocas de coleta, com três repetições, cada repetição resultou da média de duas plantas. A primeira coleta foi realizada aos 47 d.a.t. e as demais em intervalos de 21 dias.

Em cada coleta, após a determinação da área de todas as lâminas foliares, em medidor de área foliar modelo LI 3100 da Li-Cor, os diferentes órgãos das plantas foram separados em raízes, caules e folhas (lâminas foliares e pecíolos), acondicionados em sacos de papel e secos em estufa de circulação forçada de ar à temperatura de $70^{\circ} \mathrm{C}$ até que se obtivesse massa constante. Após a secagem, o material foi pesado em balança analítica e os resultados foram expressos em gramas.

Para a obtenção dos parâmetros fisiológicos da análise de crescimento, como a razão de área foliar (RAF) em dm² $\mathrm{g}^{-1}$, área foliar específica (AFE) em dm² $\mathrm{g}^{-1}$, taxa assimilatória líquida (TAL) em g. $\mathrm{dm}^{2}$ dia e taxa de crescimento relativo (TCR) em g g $^{-1}$ dias, as variáveis área foliar, massa seca das folhas e massa seca total das plantas foram estimadas e ajustadas em relação ao tempo, pelo programa computacional ANACRES, utilizando-se a equação exponencial quadrática, de acordo com as especificações de PORTES \& CASTRO JÚNIOR (1991). Esses parâmetros fisiológicos, por serem dados calculados, não obedecem as pressuposições básicas para a análise de variância. Portanto, os dados foram apresentados na forma de gráfico avaliando-se o comportamento das curvas.

\section{RESULTADOS E DISCUSSÃO}

A variação da razão de área foliar de Salvia officinalis L., submetida aos diferentes tratamentos com reguladores de crescimento vegetal, nas várias coletas, pode ser observada na figura 1-A. De maneira geral, todos os tratamentos, com exceção da testemunha, promoveram diminuição constante da RAF. Esses resultados assemelham-se aos de BARREIRO et al. (2006), que, estudando o efeito de reguladores vegetais no desenvolvimento de Ocimum basilicum L., observaram máxima RAF na primeira coleta, decrescendo nos períodos seguintes. Esta tendência da RAF elevada no início do ciclo vegetativo, decrescendo, posteriormente, com a maturação da planta também foi observada por outros autores (RODRIGUES et al., 1993; URCHEI \& RODRIGUES, 1994; STEFANINI et al., 1998).

As plantas não-tratadas (testemunha) apresentaram menor valor da RAF no início do ciclo e pequeno aumento dos 47 aos 68 d.a.t. e queda constante após este período. Este comportamento representa evidência de que essas plantas apresentaram crescimento mais lento em relação às tratadas com reguladores vegetais. A RAF representa a relação entre a área foliar que é responsável pela interceptação da energia luminosa e $\mathrm{CO}_{2}$ e a massa seca total resultante da fotossíntese. Assim, com o crescimento da planta, aumenta a interferência das folhas superiores, com tendência a diminuir a área útil (BENINCASA, 2003). O tratamento com BAP, uma citocinina sintética, apresentou a maior RAF nas duas primeiras coletas, que pode ser atribuída à atividade das citocininas na promoção da expansão foliar.

As plantas tratadas com $\mathrm{GA}_{3}$, Stimulate $^{\circledR}$ e a testemunha apresentaram queda constante na AFE ao longo do ciclo de desenvolvimento (Figura 1-B). Já nas plantas tratadas com BAP e ethephon, observouse queda na AFE até aos 110 dias após o transplante, ocorrendo pequeno aumento após este período. Isso pode ter ocorrido em razão da alteração morfológica nas folhas, pelo aumento na expansão foliar, já que o BAP promove aumento na expansão foliar e na formação do aparelho fotossintético (TAIZ \& ZEIGER, 2002). Assim, quando novas folhas se desenvolvem, a AFE volta a aumentar, o que expressa menor espessura. Tal comportamento é esperado em plantas em que os reguladores vegetais estimulam o crescimento vegetativo.

No início do desenvolvimento, os valores da AFE podem ser maiores, revelando folhas pouco espessas, com pouca massa seca e área foliar. Com o desenvolvimento da planta, aumenta-se a área foliar e a massa seca das folhas, tendendo à queda dos valores dessa variável (BENINCASA, 2003). Aárea foliar é um componente morfofisiológico e a massa um componente anatômico de uma espécie vegetal, pois está relacionado à composição interna (número e tamanho) das células do mesofilo. A partir disso infere-se que o inverso da AFE reflete a espessura das folhas (BENINCASA, 2003). FERREIRA (1996) relata que decréscimos na AFE indicam aumento na espessura da folha resultante do aumento e do tamanho do número de células nas plantas.

As plantas testemunha apresentaram valores iniciais altos para a TAL, ocorrendo decréscimo até aos 89 d.a.t. e pequeno aumento após 110 dias (Figura 1-C). O tratamento com $\mathrm{GA}_{3}$ apresentou aumento da TAL no decorrer do ciclo, enquanto que com BAP e ethephon as plantas apresentaram aumentos até aos 89 e 68 d.a.t., respectivamente, e queda após estes períodos. Já plantas tratadas com Stimulate $^{\circledR}$, mantiveram-se constantes até aos 68 d.a.t. e apresentaram diminuição após este período.

A taxa assimilatória líquida diminui com a idade das plantas por sombreamento das folhas interiores (WATSON, 1952; MILTHORPE \& MOORBY, 1974). Os resultados verificados no presente trabalho estão parcialmente em concordância com os resultados 
A

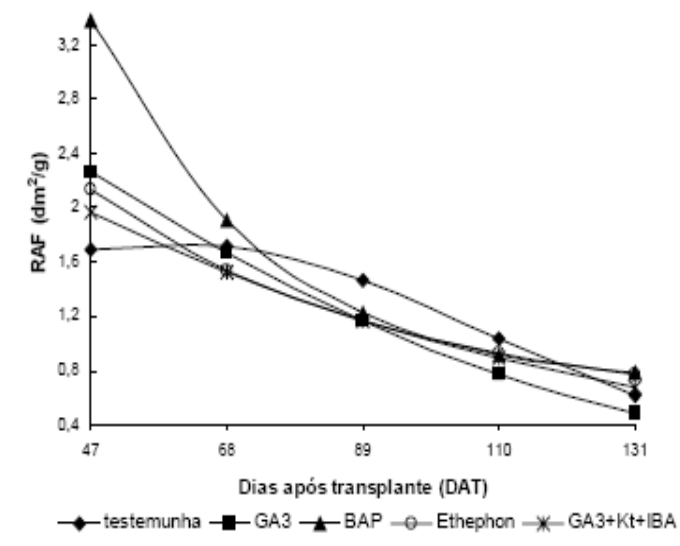

\begin{tabular}{|c|c|c|}
\hline Tratamentos & Equacões & $r^{2}$ \\
\hline & $\mathrm{Y}=0,016 \mathrm{e} 0,076 \mathrm{x}-0,0002 \mathrm{x}^{2}$ & 0,9853 \\
\hline \multirow[t]{2}{*}{ Testenuma } & $Z=0,013$ e $0,100 x-0,0004 x^{2}$ & 0,9901 \\
\hline & $\bar{Y}=0,047 e 0,061 \mathrm{x}-0,0002 \mathrm{x}^{2}$ & 0,9970 \\
\hline \multirow[t]{2}{*}{$\mathrm{GA}_{3}$} & $Z=0,175$ e $0,054 x-0,0002 x^{2}$ & 0,9756 \\
\hline & $\mathrm{Y}=0,008 \mathrm{e} 0,096 \mathrm{x}-0,0003 \mathrm{x}^{2}$ & 0,9990 \\
\hline \multirow[t]{2}{*}{ BAP } & $Z=0,162$ e $0,051 x-0,0002 x^{2}$ & 0,9878 \\
\hline & $\mathrm{Y}=0,007 \mathrm{e} 0,098 \mathrm{x}-0,0003 \mathrm{x}^{2}$ & 0,9981 \\
\hline \multirow[t]{2}{*}{ Etheppon } & $\bar{Z}=0,038$ e $0,076 \mathrm{x}-0,0003 \mathrm{x}^{2}$ & 0,9904 \\
\hline & $\mathrm{Y}=0,018 \mathrm{e} 0,079 \mathrm{x}-0,0003 \mathrm{x}^{2}$ & 0,9997 \\
\hline $\mathrm{GA}+\mathrm{Kt}+\mathrm{BBA}$ & $\mathrm{Z}=0,062$ e $0,067 \mathrm{x}-0,0002 \mathrm{x}^{2}$ & 0,9824 \\
\hline
\end{tabular}

Y: Ajuto da masca socat total om funglio do tom:

C

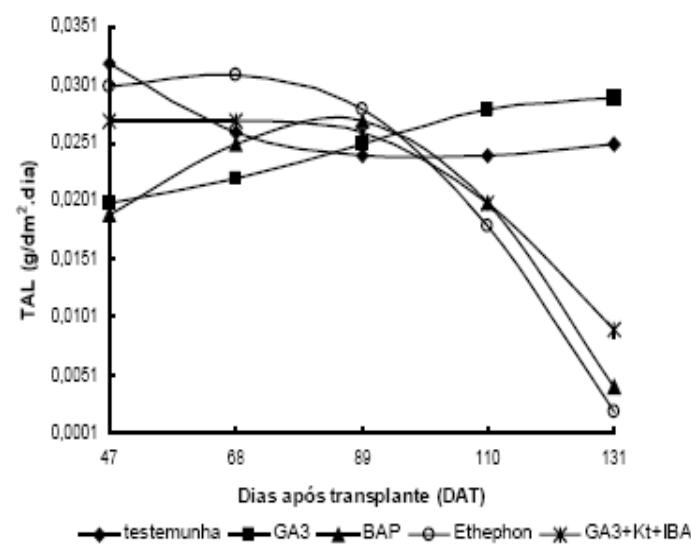

B

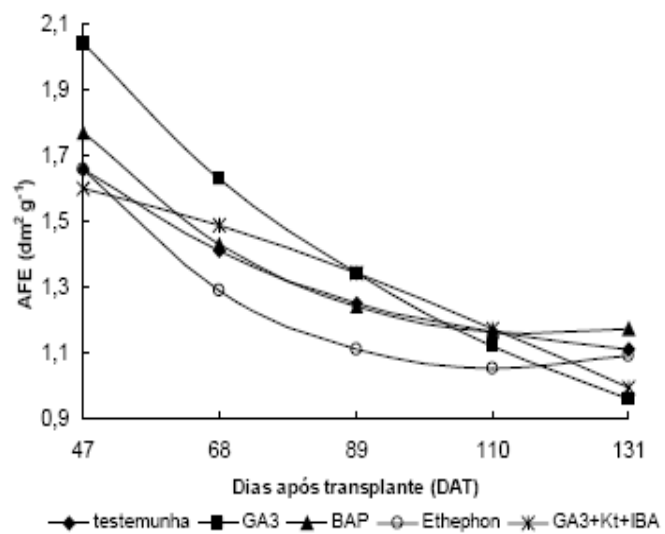

D

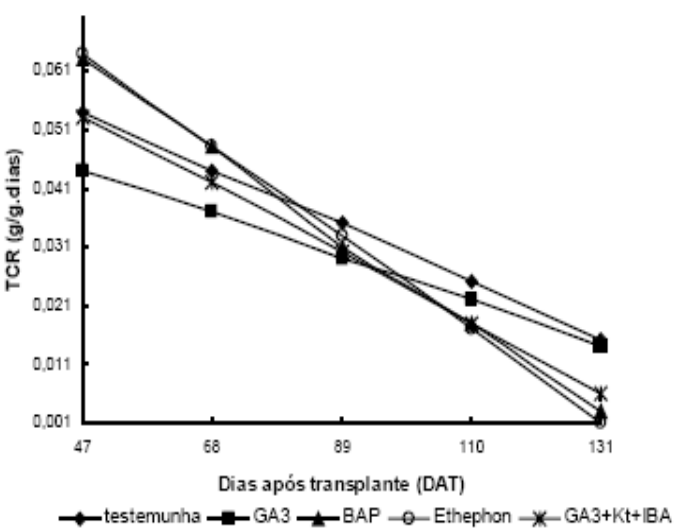

Figura 1 - Razão de Área Foliar (A), Área Foliar Específica (B), Taxa Assimilatória Líquida (C) e Taxa de Crescimento Relativo (D) de plantas de Salvia officinalis L. submetidas a tratamentos com água (testemunha), ácido giberélico (GA ( $_{3}$ ) a 100 $\mathrm{mg} \mathrm{L}^{-1}$, benzilaminopurina (BAP) a $100 \mathrm{mg} \mathrm{L}^{-1}$, etehephon a $100 \mathrm{mg} \mathrm{L}^{-1}$ e Stimulate ${ }^{\circledR}\left(\mathrm{GA}_{3}+\mathrm{Kt}+\mathrm{IBA}\right)$ a $2 \%$. Em cinco épocas de coletas, 47, 68, 89, 110 e 131 dias após transplante (d.a.t.).

Ciência Rural, v.38, n.8, nov, 2008. 
verificados por estes autores, uma vez que ocorreu aumento da TAL em plantas tratadas com $\mathrm{GA}_{3}$ ao longo do ciclo de desenvolvimento. Este regulador vegetal promoveu maior alongamento caulinar, folhas menores e mais espalhadas, evitando assim o autosombreamento e promovendo aumento na TAL.

A variação da taxa de crescimento relativo (TCR) de S. officinalis, submetida aos diferentes tratamentos com reguladores de crescimento vegetal, pode ser observada na figura 1-D. Os maiores valores para TCR, de maneira geral, foram verificados na primeira coleta, aos 47 d.a.t., com decréscimo nas coletas seguintes. A TCR das plantas tratadas com ethephon, BAP e testemunha apresentaram-se mais elevadas aos 47 d.a.t. O decréscimo da TCR ao longo do tempo também foi observado por BARREIRO et al. (2006) em plantas de Ocimum basilicum L. tratadas com reguladores vegetais.

A TCR em todos os tratamentos durante o período de estudo apresentaram comportamento semelhante, em que a fase inicial representa rápido acúmulo de material, seguida de comportamento tendendo à diminuição ao longo das coletas. Essa diminuição pode ser explicada pela elevação da atividade respiratória e pelo auto-sombreamento, cuja importância aumenta com a idade da planta.

\section{CONCLUSÕES}

Os parâmetros fisiológicos razão de área foliar, área foliar específica, taxa assimilatória líquida e taxa de crescimento relativo são influenciados pelos diferentes reguladores de crescimento vegetal. Porém, os principais efeitos são observados em plantas tratadas com BAP, que apresentam maiores valores de RAF aos 47 d.a.t. em razão do aumento na expansão foliar promovida por esta substância. Além disso, plantas tratadas com $\mathrm{GA}_{3}$, em que a TAL aumentou até aos 131 d.a.t. devido ao maior alongamento do entrenó, promovido por este regulador, evitando o sombreamento das folhas inferiores, resultou no aumento constante deste parâmetro.

\section{AGRADECIMENTOS}

Ao Conselho Nacional de Desenvolvimento Científico e Tecnológico (CNPq), pelo apoio financeiro concedido para a realização deste trabalho.

\section{REFERÊNCIAS}

BARICEVC, D.; BARTOL, T. The biological/pharmacological activity of the Salvia genus V., pharmacology. In: KINTZIOS, S.E. (Ed.). Sage: the Genus salvia. Marston: Harwood Academic, 2000. p.347-420.
BARREIRO, A.P. et al. Análise de crescimento de plantas de manjericão tratadas com reguladores vegetais. Bragantia, Campinas, v.65, n.4, p.563-567, 2006.

BENINCASA, M.M.P. Análise de crescimento de plantas: noções básicas. 2.ed. Jaboticabal: FUNEP, 2003. 41p.

COSTA, A.F. Farmacognosia. Lisboa: Fundação Calouste, 1994. 1031p.

CUVELIER, M.E. et al. Antioxidative activity and phenolic composition of pilot-plant and commercial extracts of sage and rosemary. Journal of the American Oil Chemists' Society, Champaign, v.73, p645-652, 1996.

EVANS, W.C. Farmacognosia. México: Interamericana/ Magraw-Hill, 1991. 812p.

FERREIRA, E. Ajustamento osmótico e análise de crescimento de plantas de milho (Zea mays L.), em função do nível de potássio e estresse hídrico. 1996. 112f. Tese (Doutorado em Botânica) - Curso de Pós-graduação em Ciências Biológicas, Universidade Estadual Paulista, Botucatu.

HERTWIG, I.F.V. Plantas aromáticas e medicinais. São Paulo: Icone. 1991. 414p.

MAGALHÃES, A.C.N. Análise quantitativa de crescimento. In: FERRI, M.G. Fisiologia vegetal. São Paulo: EDUSP, 1986. V.1, p.331-350.

MARTINS, E.R. Plantas medicinais. Viçosa: UFV, 1998. 220p.

MILTHORPE, F.L.; MOORBY, J. Some aspects of overral growth and its modification. In: MILTHORPE, F.L.; MOORBY, J. An introduction to crop physiology. London: Cambridge University, 1974. 202p.

PORTES, T.A.; CASTRO JUNIOR, L.G. Análise de crescimento de plantas: Um programa computacional auxiliar. Revista Brasileira de Fisiologia Vegetal, Lavras, v.3, p.53-60, 1991

RODRIGUES, J.D. et al. Diferentes níveis de cálcio e o desenvolvimento de plantas de estilosantes (Stylosanthes guyanensis (AUBL.) CW “COOK”). Scientia Agrícola, Piracicaba, v.50, p.166-175, 1993.

SHUKLA, A.; FAROOQI, A.H.A.E. Review: utilization of plant growth regulators in aromatic plant production. Current Research Medicinal \& Aromatic Plants, Amsterdam, v.12, p.152-177, 1990.

STEFANINI, M.B. et al. Efeito do ácido giberélico, CCC e ethephon no conteúdo de biomassa e rendimento de óleo essencial em diferentes épocas de aplicação em Lippia Alba (Mill.) N.E.Br.-Verbenaceae. Revista Brasileira de Plantas Medicinais, Botucatu, v.1, n.1, p.39-48, 1998.

TAIZ, L., ZEIGER, E. Plant physiology. 3.ed. Sunderland: Sinauer Associates, 2002.

URCHEI, M.A.; RODRIGUES, J.D. Efeitos de potenciais de água no solo, em diferentes estádios fenológicos da cultura da cevada (Hordeum vulgare L.). Scientia Agrícola, Piracicaba, v.51, p533-540, 1994.

WATSON, D.J. The physiological basis on variation in yield. Advances in Agronomy, Newark, v.4, p.101-145, 1952. 